\title{
SITUACIÓN Y PERSPECTIVAS DE LA TRANSFERENCIA DE FUNCIONES FORESTALES A LOS GOBIERNOS REGIONALES ${ }^{1}$
}

\author{
Giuliana Nathalie Zamora Velazco \\ Investigadora independiente \\ ORCID: 0000-0002-7760-6663
}

\begin{abstract}
Resumen: El proceso de transferencia de funciones forestales, que inició en el año 2009, no se concluyó, encontrándose pendiente que quince gobiernos regionales asuman las funciones de vigilancia y control, así como de administración y fiscalización de los recursos forestales y de fauna silvestre (RFFS), contempladas en la Ley Orgánica de Gobiernos Regionales (artículo 51). Esta situación se debe a debilidades propias del proceso de descentralización a nivel nacional, aunado a la débil rectoría del SERFOR para liderar el proceso de descentralización y promover la gestión descentralizada de los RFFS, así como al poco interés de los gobiernos regionales en asumir dichas funciones. Esto originó una organización poco clara y cambiante, débil capacidad operativa y fortalecimiento de capacidades, entre otros problemas que siempre han afectado al sector forestal; es el caso de la deforestación y la corrupción asociada al aprovechamiento ilegal de recursos forestales.

Adicionalmente, se presenta un estudio de caso desde la perspectiva de cuatro departamentos. Dos de ellos, Cajamarca y Cusco, en los que aún no se ha efectuado la transferencia de funciones forestales y se mantiene una administración dependiente del SERFOR a través de una Administraciones Técnicas Forestales y de Fauna Silvestre (ATFFS). Los dos restantes, Loreto y Ucayali, en los que se efectuó la transferencia de funciones forestales, se administran en una gestión descentralizada a través de Autoridad Regional Forestal y de Fauna Silvestre (ARFFS). Como resultado, se evidenció que ambos tipos de administración presentan debilidades. En el caso de las ATFFS, presentan debilidades que radican en el poco interés del SERFOR en fortalecer su gestión, cuentan con un escaso presupuesto, incipiente participación en el presupuesto por resultados y proyectos de inversión que SERFOR ejecuta. Las ARFFS presentan debilidades
\end{abstract}


relacionadas a los escasos recursos económicos que destinan los gobiernos regionales en la administración de los RFFS y la limitada ejecución de proyectos de inversión.

Palabras clave: Transferencia de funciones, Rectoría, Deforestación, Corrupción, Debilidades, Perú.

\section{Status and Prospects of the Transfer of Forest Functions to Regional Governments}

Abstract: The process of the transfer of forestry functions, which began in 2009, was not completed; 15 Regional Governments have yet to assume the functions of monitoring and control; as well as administration and control of the forestry and wildlife resource (RFFS acronym in Spanish), provided for in the Organic Law of Regional Government (Article $51^{\circ}$ ). This situation is due to weaknesses inherent in the decentralization process at the national level, coupled with the weak leadership of SERFOR to lead the decentralization process and promote the decentralized management of RFFS, and, to the little interest of the Regional Governments in assuming these functions. This led an unclear and changing organization, weak operational capacity and capacitybuilding; among other problems, which have always affected the forest sector; this is the case with deforestation and corruption associated with harvesting.

In addition, a case study is presented from the perspective of four departments; two of them, Cajamarca and Cusco, in which the transfer of forestry functions has not yet been carried out, and an administration dependent on SERFOR is maintained through an Technical Forest and Wildlife Administrations (ATFFS acronym in Spanish). The remaining two, Loreto and Ucayali, where the transfer of forestry functions took place, are managed in a decentralized manner through an ARFFS. As a result, it was evident that both types of administration have weaknesses. In the case of the ATFFS, they have weaknesses that lie in the little interest of SERFOR in strengthening its management, they have a low budget, incipient participation in the budget for results and investment projects that SERFOR executes. The Regional Forest and Wildlife Authority 
(ARFFS acronym in Spanish) have weaknesses related to the limited financial resources allocated by the Regional Governments in the administration of RFFS and the limited implementation of investment projects.

Keywords: Transfer of Functions, Leadership, Deforestation, Corruption, Weaknesses, Peru.

\section{Giuliana Nathalie Zamora Velazco}

Magister Scientiae en Bosques y Gestión de Recursos Forestales por la Universidad Nacional Agraria La Molina. Especialización en Gestión Pública por la Escuela de Gobierno y Políticas Públicas de la PUCP. Posgrado en Objetivos de Desarrollo Sostenible por el Centro Agronómico Tropical de Investigación y Enseñanza (CATIE). Ingeniera Forestal por la Universidad Nacional Agraria La Molina. Especialista en control gubernamental del medio ambiente y recursos naturales. Actualmente ejerce la Supervisión del Órgano de Control Institucional del Servicio Nacional de Áreas Naturales Protegidas por el Estado.

Correo: giuliana.zamorav@gmail.com 


\section{Introducción}

La transferencia de competencias y funciones sectoriales a los gobiernos regionales es una de las etapas más importantes del proceso de descentralización. Esta etapa consiste en el traslado de competencias, funciones y responsabilidades desde los sectores del gobierno nacional hacia los gobiernos subnacionales.

En tal sentido, las funciones en materia agraria correspondientes a la gestión de los recursos forestales y de fauna silvestre (RFFS) debieron ser transferidas desde el año 2002, por parte de las Administraciones Técnicas Forestales y de Fauna Silvestre (ATFFS), órganos desconcentrados de la Ex Dirección General Forestal y de Fauna Silvestre, del Ministerio de Agricultura, hacia los gobiernos regionales, para que estos se constituyan como Autoridad Regional Forestal y de Fauna Silvestre (ARFFS).

Los procesos de transferencia no se concluyeron, toda vez que existen gobiernos regionales que aún no ejercen las funciones correspondientes a la administración y control de los RFFS, manteniéndose algunas ATFFS como organismos desconcentrados del Servicio Nacional Forestal (SERFOR) ${ }^{2}$. De otro lado, existen gobiernos regionales que a la fecha se han constituido como ARFFS a través de programas, direcciones ejecutivas u otras instancias.

Cabe agregar que este proceso, inconcluso a nivel nacional, configura una autoridad forestal heterogénea. Lo señalado, sumado a la corrupción que afecta al sector forestal, no hace más que agravar la problemática, la cual está estrechamente vinculada a un conjunto de actividades ilegales. Interpol (2016) ha estimado que al año 2016 el costo anual de la corrupción en el sector forestal estuvo en el orden de los 29 millones de dólares. 


\subsection{Estado de la transferencia de funciones forestales}

La reforma constitucional sobre descentralización, Ley 27680 (Congreso de la República 2002a), y la Ley de Bases de la Descentralización, Ley 27783 (Congreso de la República 2002b), son el marco general de la reforma descentralista del Estado peruano.

La Ley Orgánica de Gobiernos Regionales, Ley 27867, establece como función específica de los gobiernos regionales (artículo 51):

e) Desarrollar acciones de vigilancia y control para garantizar el uso sostenible de los recursos; y, q) Otorgar permisos, autorizaciones y concesiones forestales, en áreas al interior de la región, así como ejercer labores de promoción y fiscalización en estricto cumplimiento de la política forestal nacional (Congreso de la República 2002c).

En base a esta ley, se inició el proceso de transferencia de forma progresiva hasta el año 2013, teniendo como resultado que nueve gobiernos regionales asuman las funciones forestales. El detalle de lo señalado se muestra en la tabla 1 :

Tabla 1. Gobiernos regionales que concluyeron el proceso de transferencia de funciones forestales

\begin{tabular}{c|c|c|c}
\hline $\mathbf{N}^{\circ}$ & Gobierno Regional & Resolución Ministerial & Fecha \\
\hline 1 & Loreto & $\mathrm{N}^{\circ} 0793-2009-\mathrm{AG}$ & $12 / 11 / 2009$ \\
\hline 2 & San Martin & $\mathrm{N}^{\circ} 0792-2009-\mathrm{AG}$ & $12 / 11 / 2009$ \\
\hline 3 & Ucayali & $\mathrm{N}^{\circ} 0019-2010-\mathrm{AG}$ & $29 / 01 / 2010$ \\
\hline 4 & Madre de Dios & $\mathrm{N}^{\circ} 0301-2010-\mathrm{AG}$ & $30 / 04 / 2010$ \\
\hline 5 & Amazonas & $\mathrm{N}^{\circ} 0696-2010-\mathrm{AG}$ & $08 / 11 / 2010$ \\
\hline 7 & La Libertad & $\mathrm{N}^{\circ} 0303-2011-\mathrm{AG}$ & $21 / 07 / 2011$ \\
\hline 8 & Tumbes & $\mathrm{N}^{\circ} 0170-2012-\mathrm{AG}$ & $11 / 05 / 2012$ \\
\hline 9 & Ayacucho & $\mathrm{N}^{\circ} 0291-2012-\mathrm{AG}$ & $17 / 08 / 2012$ \\
\hline
\end{tabular}

Fuente: Resoluciones Ministeriales del MINAGRI, elaborado por Zamora Velazco (2020). 
De acuerdo a lo expuesto, nuestro país cuenta con dos tipos de administración y control de los RFFS; por una parte, se aplica un modelo desconcentrado con trece ATFFS en el ámbito de quince gobiernos regionales; y en otros nueve departamentos se aplica un modelo descentralizado, en los que la administración y control de los RFFS están a cargo de las ARFFS.

\section{Figura 1. Tipos de administración de los RFFS a nivel nacional}

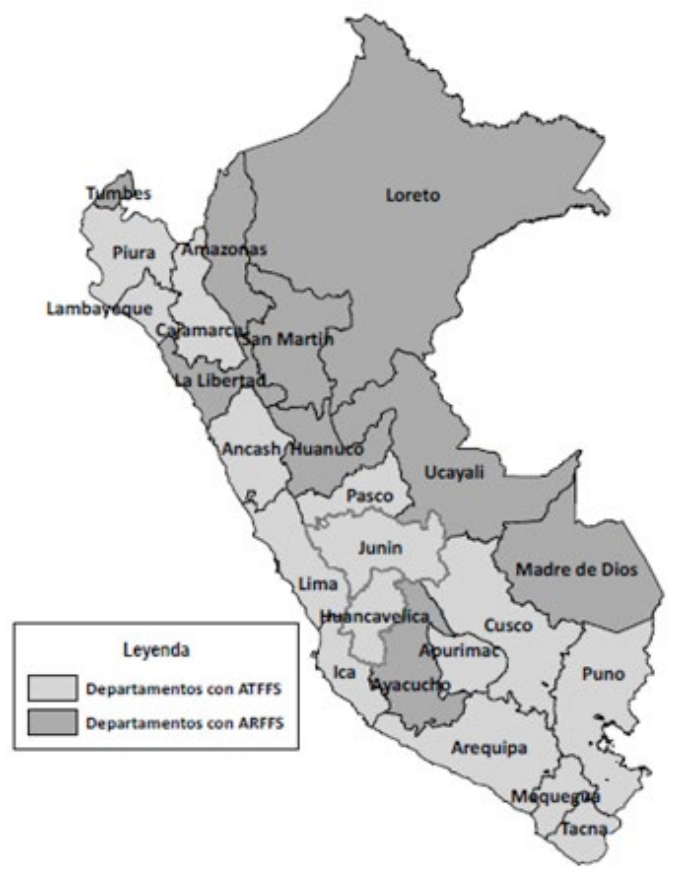

Fuente: Resoluciones Ministeriales del MINAGRI, elaborado por Zamora Velazco (2020).

Al respecto, el Informe Anual de Descentralización a junio de 2018, elaborado por la Secretaría de Descentralización de la Presidencia del Consejo de Ministros (PCM), reportó un avance del 94\% de la transferencia de funciones desde el gobierno nacional a los gobiernos regionales (PCM 2018).

A nivel sectorial, al 2019 el MINAGRI, hoy Ministerio de Desarrollo Agrario y Riego, reportaba un avance del $92 \%$ en la transferencia de las funciones en materia agraria (artículo 51) a los gobiernos regionales (MINAGRI 2019). 


\section{Factores que explican la inconclusa transferencia de funciones forestales}

\subsection{Las debilidades propias del proceso de descentralización del Perú}

Según PCM (2017), la Reforma Constitucional dispuso que la descentralización se realizaría por etapas, en forma progresiva y ordenada, conforme a criterios que permitieran una adecuada asignación de competencias y transferencia de recursos del gobierno nacional hacia los gobiernos regionales y locales, a través de las etapas dispuestas por la Ley de Bases de la Descentralización. Sin embargo, lo señalado no se cumplió, generándose las debilidades que se listan a continuación:

- El proceso de transferencia de funciones fue acelerado, no se determinó la capacidad de gestión con la que contaban los gobiernos regionales antes de que reciban y ejerzan las funciones a transferirse (CGR 2014).

- No se desarrollaron capacidades de los gobiernos regionales a lo largo del proceso de descentralización (ProDescentralización 2013).

- La escasa articulación existente entre los gobiernos regionales y locales (PCM 2012).

- El déficit significativo en la relación intergubernamental entre los sectores y los gobiernos subnacionales (ProDescentralización 2016).

- El presupuesto público continúa privilegiando a las entidades del gobierno nacional y limitando los recursos a los gobiernos subnacionales (MEF 2019a).

Figura 2. Distribución del presupuesto devengado por SERFOR (Lima y otros departamentos)

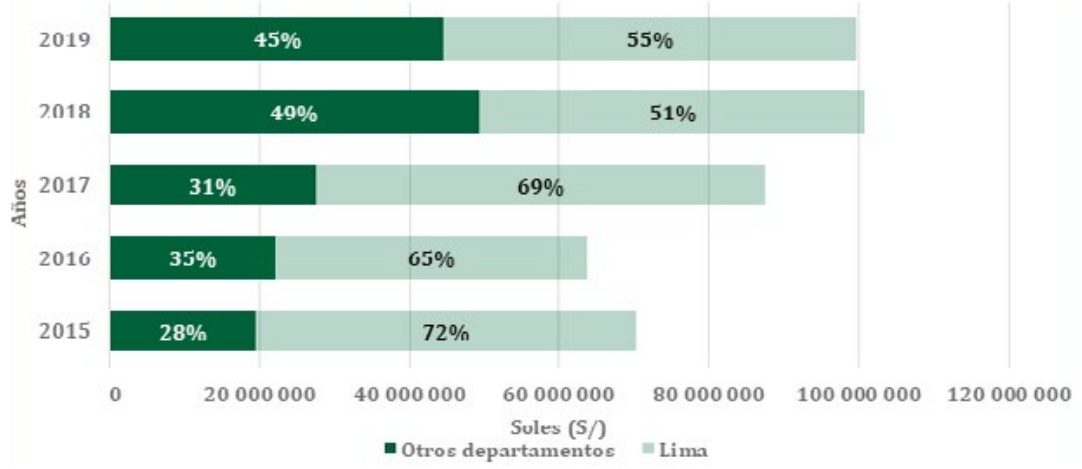

Fuente: Portal de Transparencia Económica-Consulta Amigable (MEF 2019a), elaborado por Zamora Velazco (2020). 
Además de lo señalado, la Secretaría de Descentralización, el órgano articulador y de enlace entre el gobierno nacional y los gobiernos regionales y locales, no cuenta con autonomía técnica, administrativa y económica para el ejercicio de sus funciones, ya que está supeditada funcionalmente a la gestión administrativa y económica de la PCM (CGR 2014).

\subsection{La débil rectoría del SERFOR para liderar el proceso de descentralización}

Según ProDescentralización (2016), la débil rectoría de los sectores en el proceso de descentralización dificultó los mecanismos de asignación de responsabilidades, la limitada articulación intergubernamental y multisectorial, y la falta de acompañamiento a los gobiernos regionales para fortalecer capacidades. Este es el caso del Ministerio de Agricultura, hoy Ministerio de Desarrollo Agrario y Riego, que a través del SERFOR ha dado poca claridad en cuanto al carácter temporal de las ATFFS —en el marco normativo vigente-, así como una política sectorial que no define acciones concretas respecto a la gestión descentralizada de los RFFS.

Para contextualizar lo señalado, en julio de 2011, mientras se encontraba en proceso la transferencia de funciones a los gobiernos regionales, se aprobó la Ley Forestal y de Fauna Silvestre, Ley 29763, que modificó la estructura de la autoridad forestal, estableciendo la organización del sector forestal (Congreso de la República 2011). Sin embargo, no se contempló en ese dispositivo legal qué ocurriría con las ATFFS, en tanto se culmine el proceso de transferencia de funciones a los gobiernos regionales, y no se establecieron plazos para dicha transferencia, ni otra condicionante que establezca que el SERFOR en su rol rector tenga que liderar este proceso, fortalecer relaciones intergubernamentales y las capacidades de los gobiernos regionales.

En el año 2013, se aprobó el primer Reglamento de Organización y Funciones (ROF) del SERFOR, el cual estableció que hasta que se concluya el proceso de transferencia de funciones a los gobiernos regionales, las ATFFS se incorporan al SERFOR, con las funciones establecidas en el Decreto Supremo 010-2009-AG (MINAGRI 2013).

En tal sentido, la Ley Forestal 29763 y el ROF del SERFOR no contemplaron la existencia de las ATFFS en el largo plazo; asimismo, tampoco resaltaron el rol que como ente rector le correspondía al SERFOR a fin de asegurar que el proceso de transferencia de funciones a los gobiernos regionales se realice; ello, primordialmente, para promover la gestión descentralizada de los RFFS y darle cumplimiento a la organización que establece la Ley Forestal vigente. 
Otras debilidades de la Ley 29763 fueron reveladas por Dourojeanni (2013), que refiere:

Se crea un SERFOR con una autonomía envidiable y correctamente, se le da un Consejo Directivoy una Comisión Nacional Forestal consultiva. Pero, al mismo tiempo, la ley determina una descentralización radical, dejando a la «Autoridad Forestal Nacional», es decir el SERFOR, con muy pocas funciones ejecutivas y casi ninguna autoridad.

Asimismo, Dourojeanni (2019) denota el pobre aporte de la Política Nacional Forestal, señalando que no contiene ninguna propuesta concreta ni estrategia para asegurar que su propósito sea realizable; es decir, no concreta una postura respecto a diversos temas, entre ellos, la gestión descentralizada de los RFFS.

\subsection{El poco interés de los gobiernos regionales en recibir las funciones forestales}

El Sector Agricultura informó en su «Plan de Transferencia de Competencias Sectoriales a los Gobiernos Regionales y Gobiernos Locales», del año 2017, que SERFOR solicitó a los quince gobiernos regionales pendientes de recibir las funciones forestales la adecuación de sus instrumentos de gestión, Texto Único de Procedimientos Administrativos (TUPA) y Reglamento de Organización de Funciones (ROF), incorporando las funciones que asumirían.

Al respecto, al 31 de diciembre de 2018, se informó que solo el Gobierno Regional de Pasco presentó dos instrumentos de gestión, y el Gobierno Regional de Junín solo el ROF; por otro lado, los Gobiernos Regionales de Tacna, Piura, Cajamarca, Lambayeque, Ica y Puno solo solicitaron la transferencia de funciones, pero no adecuaron sus procedimientos a la normatividad forestal vigente (MINAGRI 2019).

En 2019, el MINAGRI aprobó su «Plan de Transferencia de Competencias Sectoriales a los Gobiernos Regionales y Gobiernos Locales», en el cual informó que la transferencia de funciones forestales, literales e) y q) del artículo 51 de la Ley Orgánica de Gobiernos Regionales, continúa pendiente para quince gobiernos regionales (MINAGRI 2019).

Lo señalado revela un pobre interés en recibir las funciones forestales por parte de estos quince gobiernos regionales al dilatar acciones administrativas, como la adecuación de documentos de gestión (TUPA y ROF), que únicamente implican toma de decisiones, lo cual deja entrever que este proceso, al depender enteramente de los gobiernos regionales, no se realizará en el corto plazo. 


\section{Problemas en la gestión de los RFFS en el marco de la transferencia de funciones forestales}

A continuación, se resumen estudios que han incidido en la problemática del sector forestal.

\subsection{Deforestación}

En el territorio nacional, el proceso de deforestación ha incrementado en las últimas décadas; por ejemplo, el documento Mapa Forestal del Perú (Malleux 1975) del año 1975 informaba respecto a un área deforestada de 4500000 ha, mientras que en el año 1995 el Mapa Forestal del Perú (INRENA 1996) señalaba una deforestación ascendente a 6948237 ha; y al año 2000, el documento Mapa de Deforestación de la Amazonia Peruana informaba respecto a una deforestación de 7172553 ha (MINAM 2009).

La publicación Cuantificación y análisis de la deforestación en Amazonía Peruana en el periodo 2010-2014, señala que en el periodo 2000-2005 la superficie de pérdidas fue de 329005 ha, y en el periodo 2005-2010 fue de 571390 ha. Asimismo, en el periodo 2010-2014 la conversión de la cobertura de bosques a otros usos fue de 415278 ha, con una tasa de deforestación de 103819 ha/año. Siendo que el total de área deforestada en los últimos catorce años fue de 1315674.67 ha (MINAM 2015a). El detalle se muestra en la figura siguiente:

\section{Figura 3. Evolución de las pérdidas de bosques por deforestación de 2000 a 2014}

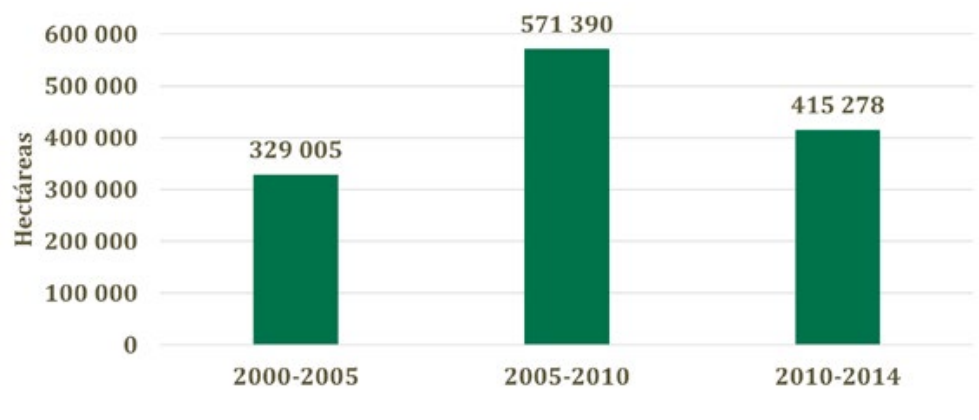

Fuente: MINAM (2015a), elaborado por Zamora Velazco (2020).

Si bien la figura anterior aparentemente indica que la deforestación en el último periodo analizado disminuyó (2010-2014), según Geobosques (2019), que cuenta con datos de pérdida anual desde 2001-2019, se tiene que desde el 
año 2013 al 2018 la deforestación consecutivamente ha superado las 150000 hectáreas por año, siendo el máximo de deforestación alcanzada en el año 2014 con 177566 hectáreas.

Bajo esa óptica, es pertinente mostrar cómo se comportó la deforestación en función a la descentralización de funciones forestales, en la figura 4 se dividen las barras de deforestación en colores que diferencian los años previos al inicio de la descentralización (2001-2008) y los posteriores al inicio del proceso (2009-2019).

Figura 4. Pérdida anual de bosques previo y durante la transferencia de funciones forestales

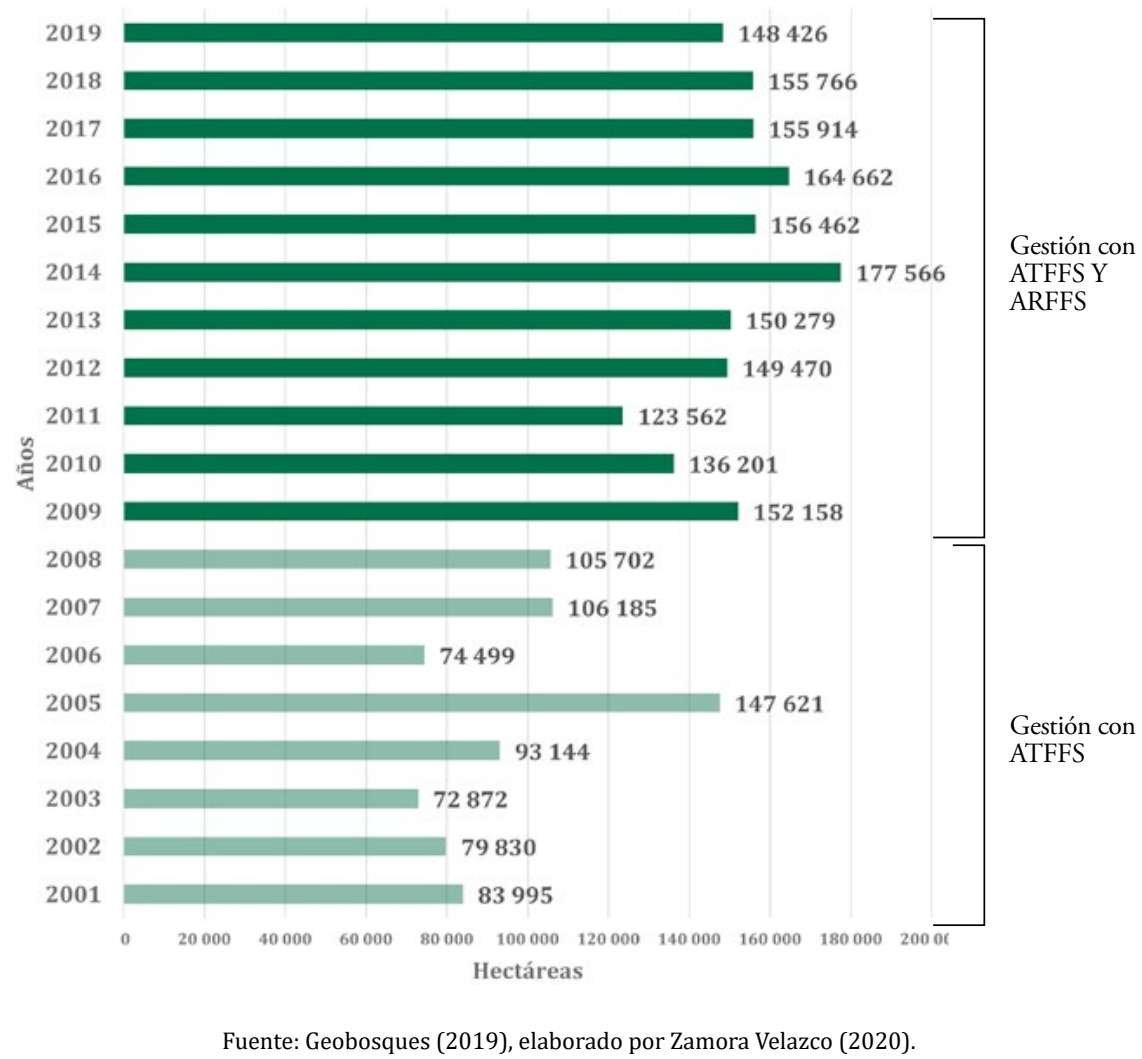

Como se observa en la figura 4, la deforestación presenta un claro incremento desde que inició el proceso de transferencia de funciones forestales, la cual se realizó inicialmente en los departamentos de Loreto y San Martín en 2009, seguidos de Madre de Dios y Ucayali en 2010, departamentos amazónicos con la mayor cobertura de bosques naturales del Perú. 


\subsection{Corrupción}

A continuación, se muestran diversas publicaciones que dejaron al descubierto prácticas corruptas en el sector forestal peruano.

- El informe La máquina lavadora - Cómo el fraude y la corrupción en el sistema de concesiones están destruyendo el futuro de los bosques de Perú, concluyó que el MINAGRI no estaba controlando de manera adecuada las actividades de las concesiones; que el MINAM no estaba monitoreando de modo eficiente las actividades de taladores ilegales en las áreas protegidas; y que los gobiernos regionales aún no estaban en capacidad de cumplir una labor eficaz para impedir las ilegalidades en el campo ni dar seguimiento legal a los casos que surjan (EIA2012).

- En el año 2014 el OSINFOR efectuó la supervisión a 144 títulos habilitantes en el marco de la Operación Amazonas. Producto de dicha fiscalización,seconstatóencampolainexistenciade8208árboles, cuyo supuestoaprovechamientofueaprobadoatravésdePlanesOperativos Anuales (POA) ${ }^{4}$ en las regiones de Loreto (64\%), Ucayali (34\%) y Huánuco (2\%); siendo que la mayor cantidad de árboles inexistentes declarados en los POA correspondían a permisos forestales en comunidades nativas (39\%), seguido de permisos forestales en predios privados (36\%) y concesiones forestales maderables (25\%) (OSINFOR 2015).

- La publicación «Descubriendo los riesgos de corrupción en el sector forestal ${ }^{5}$ hizo menciónal caso del exalcalde dela ProvinciadeCoronel Portillo, Luis Valdez Villacorta, quien fue arrestado por estar envuelto en tráfico de drogas a través de embarcaciones que transportaban madera contraplacada. El exalcalde controlaba una red de sobornos a funcionarios para movilizar madera ilegal fuera del país. Utilizando esta red, el exalcalde y otros traficantes de drogas pudieron mover la cocaína escondida en los envíos de madera contraplacada. Tras el arresto, la Policía encontró activos valorados por el monto de 71 millones de dólares cuya procedencia no pudo ser explicada (Interpol 2016).

- Global Witness (2019) ha sido claro en afirmar que el fracaso de los gobiernos regionales fomenta la tala ilegal, facilitando el blanqueo de madera ilegal al no inspeccionar los títulos habilitantes y al informar tarde al OSINFOR de las nuevas zonas de tala, o no informar en absoluto. 
Asimismo, comprobó que los POA no se inspeccionan adecuadamente, no se verifican datos como geolocalización ni identificación de los árboles a extraerse. Sus resultados revelaron que en Loreto más del $50 \%$ de los POA contenían 10\% o más de árboles inexistentes, mientras que en Madre de Dios el porcentaje era próximo al 42\% y en Junín al 25\%.

\section{Perspectivas de la gestión de los RFFS}

\subsection{Perspectiva de la gestión desconcentrada: caso Cajamarca y Cusco}

Dos departamentos que continúan con una gestión desconcentrada de sus RFFS, a través de una ATFFS dependiente de SERFOR, y que cuentan con mayores extensiones de bosques naturales son Cajamarca y Cusco (MINAM 2015b).

La figura 5 muestra el presupuesto devengado por el SERFOR en ambos departamentos, observándose que en el periodo comprendido entre 2015 y 2019 el presupuesto que destinó SERFOR en ambos departamentos no superó el millón y medio de soles.

Figura 5. Presupuesto devengado por el SERFOR en las ATFFS Cajamarca y Cusco

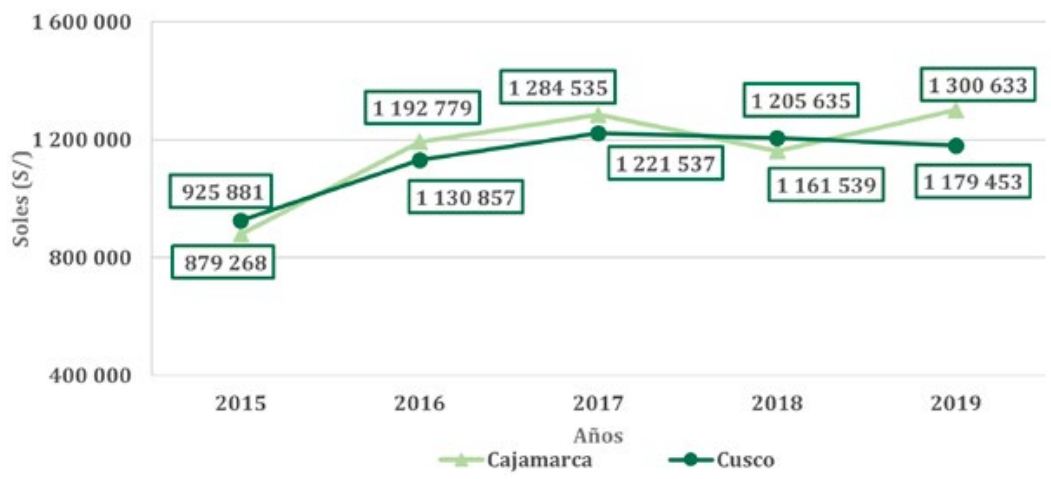

Fuente: Portal de Transparencia Económica-Consulta Amigable (MEF 2019a), elaborado por Zamora Velazco (2020).

Adicionalmente a los bajos presupuestos devengados en ambos departamentos, el SERFOR no ejecutó ningún Proyecto de Inversión (PI) en su ámbito (MEF 2019b). 
Por otro lado, la tabla 2 muestra el presupuesto devengado exclusivamente por el SERFOR bajo el Programa Presupuestal PP 130: «Competitividad y aprovechamiento sostenible de los recursos forestales y de la fauna silvestre» en las ATFFS de los departamentos de Cajamarca y Cusco, comparado con el presupuesto total del SERFOR devengado bajo dicho programa presupuestal, en el periodo 2015-2019. A continuación, se muestran los resultados obtenidos.

Tabla 2. Presupuesto devengado por el SERFOR en los PP 130 de las ATFFS Cajamarca y Cusco

\begin{tabular}{|c|c|c|c|c|c}
\hline \multirow{2}{*}{ Años } & SERFOR & \multicolumn{2}{|c|}{ Cajamarca } & \multicolumn{2}{c}{ Cusco } \\
\cline { 2 - 5 } & $\begin{array}{c}\text { Presupuesto } \\
\text { devengado en } \\
\text { el PP 130 (S/) }\end{array}$ & $\begin{array}{c}\text { Presupuesto } \\
\text { devengado en } \\
\text { el PP 130 (S/) }\end{array}$ & $\begin{array}{c}\text { \% del total } \\
\text { devengado en } \\
\text { PP 130 por el } \\
\text { SERFOR }\end{array}$ & $\begin{array}{c}\text { Presupuesto } \\
\text { devengado en } \\
\text { el PP 130 (S/) }\end{array}$ & $\begin{array}{c}\text { \% del total } \\
\text { devengado en } \\
\text { 130 por el } \\
\text { SERFOR }\end{array}$ \\
\hline $\mathbf{2 0 1 5}$ & 51329466 & 879268 & $2 \%$ & 925881 & $2 \%$ \\
\hline $\mathbf{2 0 1 6}$ & 47945377 & 1192779 & $2 \%$ & 1130857 & $2 \%$ \\
\hline $\mathbf{2 0 1 7}$ & 52589061 & 1284535 & $2 \%$ & 1221537 & $2 \%$ \\
\hline $\mathbf{2 0 1 8}$ & 81271695 & 1161539 & $1 \%$ & 1205635 & $1 \%$ \\
\hline $\mathbf{2 0 1 9}$ & 72416223 & 1300633 & $2 \%$ & 1179453 & $2 \%$ \\
\hline
\end{tabular}

Fuente: Portal de Transparencia Económica-Consulta Amigable (MEF 2019c), elaborado por Zamora Velazco (2020).

En el caso de los departamentos de Cajamarca y Cusco, el presupuesto devengado bajo el marco de resultados en el periodo 2015-2019 alcanzó como máximo el $2 \%$ del presupuesto que ejecutó el SERFOR en el PP 130: «Competitividad y aprovechamiento sostenible de los recursos forestales y de la fauna silvestre».

La baja representatividad del presupuesto orientado a resultados en los departamentos de Cajamarca y Cusco da cuenta del poco interés por parte del SERFOR en destinar recursos hacia estos departamentos en el marco del PP 130, a pesar de que esta entidad es la responsable en gestionar el PP 130 y que las ATFFS de estos departamentos aún se encuentran bajo su dependencia.

\subsection{Perspectiva de la gestión descentralizada: caso Loreto y Ucayali}

Los departamentos de Loreto y Ucayali son los que cuentan con mayor extensión de bosques naturales (MINAM 2015b), mayor extensión de bosques de producción permanente (MINAM 2015a) y mayor incidencia de ilegalidad en el aprovechamiento forestal (OSINFOR 2016); por tanto, la gestión de sus ARFFS es de interés para el presente artículo. 
Tabla 3. Presupuesto devengado del Gobierno Regional de Loreto y su ARFFS

\begin{tabular}{cccc}
\hline Año & PIM $^{6} \begin{array}{c}\text { devengado del GR } \\
\text { (S/) }\end{array}$ & $\begin{array}{c}\text { PIM devengado } \\
\text { ARFFS } \\
\text { (S/) }\end{array}$ & $\begin{array}{c}\text { \% de PIM } \\
\text { devengado } \\
\text { por la ARFF }\end{array}$ \\
\hline $\mathbf{2 0 1 5}$ & 1232246193.85 & 2713343 & $0.20 \%$ \\
\hline $\mathbf{2 0 1 6}$ & 1335200365.48 & 1763856 & $0.10 \%$ \\
\hline $\mathbf{2 0 1 7}$ & 1618271789.00 & 460108 & $0.02 \%$ \\
\hline $\mathbf{2 0 1 8}$ & 1714305285.00 & 2133838 & $0.10 \%$ \\
\hline $\mathbf{2 0 1 9}$ & 1922474076.00 & 7261424 & $0.38 \%$ \\
\hline
\end{tabular}

Fuente: Portal de Transparencia Económica-Consulta Amigable (MEF 2019a), elaborado por Zamora Velazco (2020).

Como se observa en la tabla 3, el presupuesto que devengó la ARFFS Loreto en el periodo 2015-2019 no superó el 0.5 \% del total devengado por el Gobierno Regional de Loreto. Por otro lado, a pesar de que el Gobierno Regional incrementó su presupuesto devengado en el periodo 2015-2019, esto no se vio reflejado en el presupuesto devengado por su ARFFS.

En el caso del Gobierno Regional de Ucayali (tabla 4), se observa que destina mayores recursos que el Gobierno Regional de Loreto en la gestión de su ARFFS. Sin embargo, la representatividad de lo devengado por la ARFFS de Ucayali con respecto del total devengado por el Gobierno Regional fluctuó entre 0.5 y $1 \%$ en el periodo 2015-2019.

Tabla 4. Presupuesto devengado del Gobierno Regional de Ucayali y su ARFFS

\begin{tabular}{cccc}
\hline Año & $\begin{array}{c}\text { PIM devengado del GR } \\
\text { (S/) }\end{array}$ & $\begin{array}{c}\text { PIM devengado } \\
\text { ARFFS } \\
\text { (S/) }\end{array}$ & $\begin{array}{c}\text { \% de PIM } \\
\text { devengado } \\
\text { por la ARFF }\end{array}$ \\
\hline $\mathbf{2 0 1 5}$ & 663873162 & 6567246 & $1.0 \%$ \\
\hline $\mathbf{2 0 1 6}$ & 861033491 & 7017828 & $1.0 \%$ \\
\hline $\mathbf{2 0 1 7}$ & 848814473 & 3921700 & $0.5 \%$ \\
\hline $\mathbf{2 0 1 8}$ & 861165915 & 4827261 & $0.6 \%$ \\
\hline $\mathbf{2 0 1 9}$ & 1198380458 & 7929127 & $0.7 \%$ \\
\hline
\end{tabular}

Fuente: Portal de Transparencia Económica-Consulta Amigable (MEF 2019a), elaborado por Zamora Velazco (2020).

En relación a la inversión pública, se tiene que durante el periodo 20152019 el Gobierno Regional de Loreto ejecutó ocho Proyectos de Inversión relacionados a la gestión de los RFFS. En el periodo de cinco años se devengó un monto total de S/ 6397 934. Los montos devengados por año solo superaron el millón de soles en 2015 y 2019, siendo que la representatividad fluctuó entre $1 \%$ y $0.04 \%$ en el periodo evaluado.

6 Presupuesto Institucional Modificado. 
Tabla 5. Proyectos de Inversión del Gobierno Regional de Loreto para la gestión de los RFFS

\begin{tabular}{|ccccccc}
\hline GR LORETO & 2015 & 2016 & 2017 & 2018 & 2019 & Total \\
\hline $\begin{array}{c}\text { Total devengado } \\
\text { en PI (S/) }\end{array}$ & 175160181 & 129479373 & 301692316 & 265236942 & 329253563 & 1200822375 \\
\hline $\begin{array}{c}\text { Total en PI } \\
\text { en RFFS (S/) }\end{array}$ & 1297419 & 449733 & 116875 & 995550 & 3538357 & 6397934 \\
\hline $\begin{array}{c}\text { Devengado de PI } \\
\text { en RFFS / Total } \\
\text { devengado en PI (\%) }\end{array}$ & $1 \%$ & $0.3 \%$ & $0.04 \%$ & $0.4 \%$ & $1 \%$ & - \\
\hline
\end{tabular}

Fuente: Portal de Transparencia Económica-Consulta Amigable (MEF 2019b), elaborado por Zamora Velazco (2020).

En el periodo evaluado, el Gobierno Regional de Ucayali ejecutó once proyectos relacionados a la gestión de los RFFS. En el periodo de cinco años se devengó un monto total de S/ 7345 070. A pesar de que los montos devengados por año casi siempre han superado el millón de soles, estos montos solo han representado el 2\% o 1\% del monto total devengado por el Gobierno Regional de Ucayali en Proyectos de Inversión, por año evaluado.

Tabla 6. Proyectos de Inversión del Gobierno Regional de Ucayali para la gestión de los RFFS

\begin{tabular}{|ccccccc}
\hline GR UCAYALI & 2015 & 2016 & 2017 & 2018 & 2019 & Total \\
\hline $\begin{array}{c}\text { Total devengado } \\
\text { en PI (S/) }\end{array}$ & 146712852 & 226134497 & 274742716 & 217474835 & 496039154 & 1361104054 \\
\hline $\begin{array}{c}\text { Total en PI } \\
\text { en RFFS (S/) }\end{array}$ & 1606929 & 1887721 & 1822770 & 1374858 & 652792 & 7345070 \\
\hline $\begin{array}{c}\text { Devengado de PI } \\
\text { en RFF / Total } \\
\text { devengado en PI (\%) }\end{array}$ & $1 \%$ & $1 \%$ & $1 \%$ & $1 \%$ & $0.1 \%$ & - \\
\hline
\end{tabular}

Fuente: Portal de Transparencia Económica-Consulta Amigable (MEF 2019b), elaborado por Zamora Velazco (2020).

Por otro lado, a nivel de gobiernos regionales también se ejecuta el Programa Presupuestal PP 130: «Competitividad y aprovechamiento sostenible de los recursos forestales y de la fauna silvestre»; en tal sentido, en la tabla 7 se muestra el presupuesto (PPTO) devengado en el PP 130 de los Gobiernos Regionales de Loreto y Ucayali, y su representatividad respecto al total devengado de todos los Gobiernos Regionales en el PP 130. 
Tabla 7. Presupuesto por resultados de las ARFFS de Loreto y Ucayali

\begin{tabular}{|c|c|c|c|c|}
\hline \multirow{2}{*}{ Año } & \multicolumn{2}{|c|}{ Gobierno Regional Loreto } & \multicolumn{2}{c}{ Gobierno Regional Ucayali } \\
\cline { 2 - 5 } & $\begin{array}{c}\text { PPTO devengado } \\
\text { en el PP 130 (S/) }\end{array}$ & $\begin{array}{c}\text { \% del total } \\
\text { devengado en } \\
\text { PP 130 }\end{array}$ & $\begin{array}{c}\text { PPTO devengado } \\
\text { en el PP 130 (S/) }\end{array}$ & $\begin{array}{c}\text { \% del total } \\
\text { devengado en } \\
\text { PP 130 }\end{array}$ \\
\hline $\mathbf{2 0 1 5}$ & 1374302 & $10 \%$ & 2000 & $0.01 \%$ \\
\hline $\mathbf{2 0 1 6}$ & 538232 & $4 \%$ & 2998472 & $20 \%$ \\
\hline $\mathbf{2 0 1 7}$ & 301850 & $2 \%$ & 2674005 & $19 \%$ \\
\hline $\mathbf{2 0 1 8}$ & 1449536 & $6 \%$ & 4030662 & $16 \%$ \\
\hline $\mathbf{2 0 1 9}$ & 7169176 & $17 \%$ & 8042413 & $19 \%$ \\
\hline
\end{tabular}

Fuente: Portal de Transparencia Económica-Consulta Amigable (MEF 2019c).

De la tabla 7 se observa que la ARFFS del Gobierno Regional de Loreto ha tenido un devengado irrelevante en el PP 130; a excepción del año 2019, en el cual tuvo el segundo mayor devengado en este PP, solo siendo superado por la ARFFS del Gobierno Regional de Ucayali. La ARFFS del Gobierno Regional de Ucayali demostró conocimiento de la herramienta de presupuesto por resultados, toda vez que en los años 2017 y 2019 fue el gobierno regional que mayores recursos devengó bajo esta herramienta.

\section{Conclusiones}

- El inconcluso proceso de transferencia de funciones forestales se debe a debilidades propias del proceso de descentralización a nivel nacional, lo que sumado a la débil rectoría del SERFOR para liderar el proceso de descentralización y promover la gestión descentralizada de los RFFS, así como al poco interés de los gobiernos regionales en asumir dichas funciones, le han dado un carácter permanente a trece ATFFS dependientes del SERFOR en el ámbito de quincegobiernos regionalesque aún no ejercencomo ARFFS.

- Las ATFFS de Cusco y Cajamarca presentan debilidades que radican en el poco interés del SERFOR en fortalecer su gestión, cuentan con un escaso presupuesto, incipiente participación en el presupuesto por resultados y proyectos de inversión que SERFOR ejecuta.

- Las ARFFS de Loreto y Ucayali presentan debilidades relacionadas al escaso presupuesto que destinan los gobiernos regionales en su funcionamiento y la limitada ejecución de proyectos de inversión, así como la falta de capacidad técnica, producto de debilidades propias del proceso de descentralización a nivel nacional. 
- SERFOR, como ente rector del Sistema Nacional de Gestión Forestal y de Fauna Silvestre ${ }^{7}$, debe apoyar a los gobiernos regionales en el ámbito de los departamentos donde aún funcionan las ATFFS, a fin de que culminen los procesos administrativos pendientes para efectivizar la transferencia de funciones forestales. Para ello, SERFOR tiene que fortalecer las capacidades y los mecanismos de comunicación interinstitucionaldelosgobiernosregionales, demaneraqueseejecuten exitosamente herramientas, como presupuesto por resultados o proyectos de inversión, en favor de culminar el proceso de transferencia de funciones.

\section{Referencias}

Congreso de la República del Perú (2011). Ley N² 29763. Ley Forestal y de Fauna Silvestre. Lima, 21 de julio.

Congreso de la República del Perú (2002a). Ley N²7680. Ley de Reforma Constitucional del Capítulo XIV del Título IV, sobre descentralización. Lima, 6 de marzo. Congreso de la República del Perú (2002b). Ley $N^{\circ}$ 27783. Ley de Bases de la Descentralización. Lima,17 de julio.

Congreso de la República del Perú (2002c). Ley № 27867. Ley Orgánica de Gobiernos Regionales. Lima 16 de noviembre.

CGR (Contraloría General de la República) (2014). Estudio del proceso de descentralización en el Perú. Lima, 415p.

Dourojeanni, M. (2019). Esbozo de una nueva Política Forestal Peruana. Revista Forestal del Perú, 34(1): 4-20. http://dx.doi.org/10.21704/rfp.v34i1.1244

Dourojeanni, M. (2013). Análisis Crítico de la Ley Forestal Peruana. Boletín Informativo de Derecho, Ambiente y Recursos Naturales-DAR, 20-32.

EIA (Environmental Investigation Agency) (2012). La Máquina Lavadora-Cómo el Fraude y la Corrupción en el Sistema de Concesiones están destruyendo el futuro de los bosques de Perú. Lima, 72p.

Geobosques (Plataforma del Programa Nacional de Conservación de Bosques para la Mitigación del Cambio Climático del Ministerio del Ambiente) (2019). Bosque y Pérdida de Bosque. http://geobosques.minam.gob.pe/geobosque/view/ perdida.php

Global Witness (2019). «El justiciero forestal: Por qué se debe devolver la independencia a OSINFOR y expandir sus poderes». Informe Global Witness. https://www.globalwitness.org/documents/19591/El_justiciero_forestal.pdf INRENA (Instituto Nacional de Recursos Naturales) (1996). Guía Explicativa del Mapa Forestal 1995. 144p.

Sistema funcional integrado por los ministerios y los organismos e instituciones públicas de los niveles nacional, regional y local que ejercen competencias y funciones en la gestión forestal y de fauna silvestre por los gobiernos regionales y gobiernos locales, y por los comités de gestión de bosques reconocidos (artículo 12 de la Ley 29763). 
Interpol (International Criminal Police Organization) (2016). Uncovering the Risks of Corruption in the Forestry Sector.

Malleux, J. (1975). Mapa forestal del Perú- memoria explicativa. Lima, 161p. MEF (Ministerio de Economía y Finanzas) (2019a). Consulta Amigable- Consulta de ejecución del gasto por nivel de Gobierno. Lima. http://apps5.mineco.gob.pe/transparencia/Navegador/default.aspx

MEF (Ministerio de Economía y Finanzas) (2019b). Consulta Amigable- Consulta de ejecución del gasto por pliego (producto/proyecto) y departamento. http://apps5.mineco.gob.pe/transparencia/Navegador/default.aspx

MEF (Ministerio de Economía y Finanzas) (2019c). Consulta Amigable- Consulta de ejecución del gasto por programa presupuestal y entidad. Lima. http://apps5.mineco.gob.pe/transparencia/Navegador/default.aspx MINAGRI (Ministerio de Agricultura y Riego) (2019). Resolución Ministerial № 0221-2019-MINAGRI. Aprueba el «Plan Anual de Transferencia de Competencias Sectoriales a los Gobiernos Regionales y Locales del año 2019». 21 de junio. MINAGRI (2013). Decreto Supremo № 007-2013-MINAGRI. Aprueban «Reglamento de Organización y Funciones del Servicio Nacional Forestal y de Fauna Silvestre -SERFOR». Lima. 17 de julio.

MINAM (Ministerio del Ambiente) (2015a). Cuantificación y análisis de la deforestación en la Amazonia peruana en el periodo 2010-2011-2013-2014. Lima, 107p.

MINAM (2015b). Mapa nacional de cobertura vegetal: memoria descriptiva. Dirección General de Evaluación, Valoración y Financiamiento del Patrimonio Natural. Lima, 105p.

MINAM (2009). Mapa de Deforestación de la Amazonía Peruana-2000. Lima, 94p. OSINFOR (Organismo de Supervisión de los Recursos Forestales y de Fauna Silvestre) (2016). Sistema de Información Gerencial - Volumen movilizado proveniente de extracción ilegal en los años 2011-2016. Lima. https://observatorio.osinfor.gob.pe/Estadisticas/Home/Reportes/12

OSINFOR (2015). Resultados de las supervisiones y fiscalizaciones efectuadas por el OSINFOR en el marco del Operativo Internacional "Operación Amazonas 2014". Lima, 63p.

PCM (Presidencia del Consejo de Ministros- Secretaría de Descentralización) (2018). Informe Anual del Proceso de Descentralización 2017. Lima, 111p.

PCM (Presidencia del Consejo de Ministros) (2017). Informe Anual del Proceso de Descentralización 2016. Lima, 104p.

PCM (Presidencia del Consejo de Ministros) (2012). Plan Nacional de Descentralización 2012-2016. Lima, 147p.

ProDescentralización (2016). Informe anual sobre el estado del proceso de descentralización a enero 2016. Lima, 143p.

ProDescentralización (2013). Informe anual del proceso de descentralización 2012. Lima, $126 \mathrm{p}$. 